\title{
Frequence Des Complications De L'hypertension Arterielle Chez Les Patients Suivis En Milieu Cardiologique A Parakou En 2016
}

\author{
Codjo Houetondji Leopold, \\ Dohou Serge Hugues Mahougnon, \\ Ogboni Henock,
}

Unité d'Enseignement et de Recherche en cardiologie,

Faculté de Médecine, Université de Parakou

Amegan Nicolas,

Ecole Doctorale des Sciences de la Santé,

Université d'Abomey-Calavi, Bénin

Biaou Chabi Alphonse,

Institut Régional de Santé Publique, Université d'Abomey-Calavi, Bénin

Sonou Arnaud, Adjagba Philippe,

Unité de Soins d'Enseignement et de Recherche en Cardiologie,

Faculté des Sciences de la Santé, Université d'Abomey Calavi

Alassani Adebayo,

Unité d'Enseignement et de Recherche en Médecine Interne,

Faculté de Médecine, Université de Parakou

\section{Ahoui Seraphin,}

Unité d'Enseignement et de Recherche en Néphrologie,

Faculté de Médecine, Université de Parakou

Houenassi Dedonougbo Martin,

Unité de Soins d'Enseignement et de Recherche en Cardiologie,

Faculté des Sciences de la Santé, Université d'Abomey Calavi

\section{Resume}

Introduction : Souvent asymptomatique, l'hypertension artérielle se révèle parfois par des événements cardiovasculaires graves. Ce travail vise à déterminer les complications silencieuses et/ou chroniques de l'hypertension artérielle chez les patients suivis en milieu hospitalier à Parakou. Méthodes : L'étude était transversale, analytique réalisée du 20 Mars 2016 au 20 Juillet 2016, dans les services de cardiologie des hôpitaux publiques de la ville de 
Parakou. Etaient inclus, tous les hypertendus consentants, ayant réalisé le bilan minimum de l'Organisation Mondiale de la Santé (OMS) depuis au plus trois mois. L'échantillonnage était exhaustif. Les complications silencieuses et/ou chroniques de l'HTA ont été recherchées selon les procédures standards. L'analyse des données a été faite avec le logiciel SPSS 21. Résultats : Sur les 719 patients reçus en consultation, $316(43,9 \%)$ avaient une HTA. L'âge moyen était de 55,2 $\pm 11,9$ ans, la sexe ratio de 0,6 . L'ancienneté du diagnostic de l'HTA était inférieure à 5 ans chez $61,1 \%$ des patients. L'observance thérapeutique était bonne chez $43,9 \%$ et l'HTA était mal contrôlée chez $61,1 \%$. Au moins une complication a été observée chez $70,9 \%$ des patients. Les trois premières complications chroniques étaient l'hypertrophie ventriculaire gauche échographique $(54,3 \%)$, la protéinurie $(40,7 \%)$ et la rétinopathie hypertensive $(28,1 \%)$. Après régression logistique, l'ancienneté de l'HTA $\geq 5$ ans $(\mathrm{OR}=2,9 ; \mathrm{p}=0,011)$ et l'inobservance thérapeutique $(\mathrm{OR}=3,1 ; \mathrm{p}=0,002)$ étaient indépendamment associées à la survenue de complication de l'HTA. Conclusion : Ces données montrent la gravité de l'hypertension artérielle chez les patients et le rôle déterminant de l'inobservance thérapeutique dans la survenue de ses complications.

Mots clés : Bénin, Complications, Hypertension artérielle 


\title{
Frequency of Complications of High Blood Pressure Among Patients Followed in Cardiology to Parakou in 2016
}

\author{
Codjo Houetondji Leopold, \\ Dohou Serge Hugues Mahougnon, \\ Ogboni Henock,
}

Unité d'Enseignement et de Recherche en cardiologie,

Faculté de Médecine, Université de Parakou

Amegan Nicolas,

Ecole Doctorale des Sciences de la Santé,

Université d'Abomey-Calavi, Bénin

Biaou Chabi Alphonse,

Institut Régional de Santé Publique, Université d'Abomey-Calavi, Bénin

Sonou Arnaud, Adjagba Philippe,

Unité de Soins d'Enseignement et de Recherche en Cardiologie,

Faculté des Sciences de la Santé, Université d'Abomey Calavi

\section{Alassani Adebayo,}

Unité d'Enseignement et de Recherche en Médecine Interne,

Faculté de Médecine, Université de Parakou

\section{Ahoui Seraphin,}

Unité d'Enseignement et de Recherche en Néphrologie,

Faculté de Médecine, Université de Parakou

Houenassi Dedonougbo Martin,

Unité de Soins d'Enseignement et de Recherche en Cardiologie,

Faculté des Sciences de la Santé, Université d'Abomey Calavi

\begin{abstract}
Background: High blood pressure (HBP) is often revealed by cardiovascular event, issue of undiagnosed silent complications. This study aimed at determining the prevalence of chronic complications of HBP among patients followed in cardiology at Parakou in 2016. Methods: We performed a cross sectional analytic study from March $20^{\text {th }}$ to July $20^{\text {th }}, 2016$ in the cardiology settings of Parakou. We did a systematic recruitment of all hypertensive patients who have done the hypertension minimum check up of
\end{abstract}


World Health Organization at least three months before. HBP's complications were accessed according standard guidelines. Socio-demographic data, data on hypertension, treatment and treatment compliance were recorded for each patient. The software SPSS 21 was used for statistical analysis. Results: Among 719 patients admitted, $316(43,9 \%)$ had HBP. The mean age was 55,2 $\pm 11,9$ years and the sex ratio 0,6 . The age of hypertension was <10years in $80,4 \%$, the treatment compliance was good for $43,9 \%$ and HBP was uncontrolled for $61,1 \%$. At least one complication was observed in $70,9 \%$ of patients. The most frequents were echographic left ventricular hypertrophy $(54,3 \%)$, proteinury $(40,7 \%)$ and hypertensive retinopathy $(28,1 \%)$. After logistic regression, age of $\mathrm{HBP} \geq 5$ years $(\mathrm{OR}=2,9 ; \mathrm{p}=0,011)$ and the therapeutic inobservance $(\mathrm{OR}=3,1 ; \mathrm{p}=0,002)$ were independently associated with the complication of the HBP. Conclusion: These data show the gravity of black patients' HBP which is often complicated and the crucial role therapeutic inobservance in the arisen of these complications.

Keywords: Benin, Complications, Hypertension

\section{Introduction}

En Afrique subsaharienne, l'hypertension artérielle (HTA) reste souvent longtemps méconnue et le diagnostic est fortuit ou posé à l'occasion de complications évolutives cardiovasculaires, rénales, oculaires et cérébrales (Fourcade et al, 2007). En dehors de la poussée hypertensive simple, les modes de révélation de l'HTA en milieu africain sont dominés par les accidents vasculaires cérébraux (AVC), l'hypertrophie ventriculaire gauche (HVG), l'insuffisance cardiaque, l'insuffisance coronarienne, la rétinopathie hypertensive, les artériopathies oblitérante des membres inférieurs (AOMI), la protéinurie et l'insuffisance rénale (IR). Ces complications qui émaillent souvent l'évolution de la maladie hypertensive, constituent des éléments de mauvais pronostic (Fourcade et al, 2007; Narayanan et al, 2014).

A Parakou, la prévalence de l'HTA a atteint 24\% en 2009 (Segbedji, 2009). Les études réalisées dans différents services spécialisés ont révélé une proportion élevée des complications de l'HTA en milieu hospitalier. Sonou et al. à Cotonou en 2017, ont noté lors d'une étude sur le niveau de risque cardiovasculaire des hypertendus, 1,2\% d'infarctus du myocarde; $0,8 \%$ d'angor instable ; $1,3 \%$ d'artériopathie oblitérante des membres inférieurs ; $2,9 \%$ d'insuffisance rénale ; $3,5 \%$ d'insuffisance cardiaque et $6,1 \%$ d'accident vasculaire cérébral (Sonou, 2017). L'ensemble de ces données nous a amené à nous intéresser à l'épidémiologie des complications de l'HTA à Parakou. 


\section{Matériel et méthodes}

L'étude s'est déroulée sur une période de quatre mois (21 mars 2016 au 21 juillet 2016), dans les trois services de Cardiologie de la ville de Parakou située à $420 \mathrm{~km}$ de Cotonou, capitale économique du Bénin. Il s'agit du Centre Hospitalier Universitaire Départemental du Borgou (CHUD-B), de l'Hôpital d'Instruction des Armées (HIA) et du Cabinet Cardiologique du Septentrion.

L' étude était transversale, descriptive et analytique. La population d'étude était constituée de tous les patients reçus pour HTA, consentants, qui avaient bénéficié d'un bilan minimum de l'OMS depuis au plus trois mois. Les patients n'ayant réalisé aucun examen avaient été exclus.

La variable dépendante était l'existence d'une complication de l'hypertension artérielle. Les variables indépendantes étaient constituées des données sociodémographiques, l'équilibre tensionnel, l'ancienneté de l'HTA, les autres facteurs de risque cardiovasculaire cumulés, les modalités thérapeutiques, l'observance thérapeutique, les données électrocardiographiques et échocardiographiques.

L'étude a obtenu l'approbation des autorités académiques, sanitaires et administratives de Parakou et celle du comité local d'éthique. La confidentialité des données ainsi que l'intimité des personnes ont été respectées. Les patients chez lesquels une complication avait été diagnostiquée ont été orientés vers les unités spécialisées pour une prise en charge appropriée.

Les critères de diagnostic de l'HTA étaient :

- HTA connue, documentée et traitée.

- Pression artérielle (PA) $\geq 140 / 90 \mathrm{mmHg}$ en consultation et confirmée à l'automesure selon la règle de trois avec moyenne des $\mathrm{PA} \geq 135 / 85$ $\mathrm{mm} \mathrm{Hg}$.

L'hypertrophie ventriculaire gauche a été retenue à l'électrocardiogramme (indice de Sokolow $\geq 35 \mathrm{~mm}$ et/ou indice de Cornell $\geq 20 \mathrm{~mm}$ chez la femme et $\geq 28 \mathrm{~mm}$ chez l'homme et $\mathrm{QRS}$ fins $\leq 0,10 \mathrm{~s}$ ) et à l'échographie et doppler cardiaque (masse ventriculaire gauche supérieure à $115 \mathrm{~g} / \mathrm{m}^{2}$ chez l'homme et $95 \mathrm{~g} / \mathrm{m}^{2}$ chez la femme) (Sahn, 1978). Les critères de Framingham ont été utilisés pour le diagnostic de l'insuffisance cardiaque (McKee, 1971). La suspicion d'insuffisance coronarienne a été basée sur des critères cliniques (douleur thoracique de type angineuse) et électrocardiographiques selon les critères de Minnesota Macfarlane, 2000). L'ischémie myocardique silencieuse a été définie par l'existence d'anomalies électrocardiographiques selon les critères de Minnesota en l'absence de douleur angineuse. Le diagnostic d'AVC a été posé cliniquement chez les patients ayant présenté un déficit neurologique focal de survenue brutale. La rétinopathie hypertensive a été objectivée au fond d'œil et classée en 4 stades 
selon Keith et Wagener (Binaghi, 1995). L'artériopathie oblitérante des membres inférieurs (AOMI) a été diagnostiquée lorsque l'index de pression systolique (IPS) est inférieur à 0,9 (Hirsch, 2006). Le retentissement rénal a été retenu devant l'existence d'une protéinurie ou d'une insuffisance rénale. La proteinurie a été recherchée à la bandélette urinaire ou au dosage de la protéinurie des 24 heures. Le diagnostic de l'insuffisance rénale a été posé lorsque le débit de filtration glomérulaire était inférieur à 90 $\mathrm{ml} / \mathrm{mn} / 1,73 \mathrm{~m}^{2}$ (Levy, 2006).

Les données recueillies ont été enregistrées et analysées avec le logiciel SPSS version 21. Les variables quantitatives ont été exprimées en moyenne \pm écart-type, et les variables qualitatives en effectif et pourcentage. L'étude des facteurs associés a été faite en analyse univariée et multivariée. A l'analyse univariée, la comparaison de l'existence d'une complication de l'HTA a été faite suivant les différentes modalités des variables d'exposition. Les tests de Chi-2 non corrigés et le test exact de Fisher pour les petits effectifs ont été utilisés. Pour l'analyse multivariée, la présence d'une au moins des complications de l'HTA a été considérée. Ainsi, les différentes variables associées au seuil de significativité de $5 \%$ ou moins à l'analyse univariée, ont été introduites dans un modèle de régression logistique pas à pas descendant et les Odds ratio (OR) avec un intervalle de confiance à 95\% (IC 95\%) ont été générés. La comparaison était significative pour $\mathrm{p}<0,05$.

\section{Résultats}

Au total, 719 patients ont été admis au cours de la période d'étude, dont 316 hypertendus (43,9\%). Par ailleurs, 31 patients ont été exclus. L'analyse a donc porté sur 285patients .

\section{Caractéristiques générales des patients hypertendus}

L'âge moyen des patients était de 55,2 $\pm 11,9$ ans avec des extrêmes de 23 et 90 ans. La sex-ratio (H/F) était de 0,6. Les professions libérales dominaient l'échantillon. L'ancienneté moyenne de l'HTA était de 5,7 $\pm 0,6$ ans avec des extrêmes d'une semaine et 35 ans. Le tableau I présente ces données.

Tableau I : Caractéristiques générales des patients suivis pour hypertension artérielle en milieu cardiologique à Parakou en 2016

\begin{tabular}{|c|c|c|}
\hline & Effectif & Pourcentage (\%) \\
\hline \multicolumn{3}{|l|}{ Sexe } \\
\hline Masculin & 174 & 61,1 \\
\hline Féminin & 111 & 38,9 \\
\hline \multicolumn{3}{|l|}{ Age } \\
\hline$<50$ ans & 106 & 37,2 \\
\hline Entre 50 et $70 \mathrm{ans}$ & 155 & 54,4 \\
\hline$\geq 70$ ans & 24 & 8,4 \\
\hline Profession & & \\
\hline
\end{tabular}




\begin{tabular}{|c|c|c|}
\hline Ménagères & 79 & 27,7 \\
\hline Professions libérales & 93 & 32,7 \\
\hline Fonctionnaires d'état & 65 & 22,8 \\
\hline Retraités & 48 & 16,8 \\
\hline \multicolumn{3}{|l|}{ Ancienneté de l'HTA } \\
\hline$<$ 5ans & 174 & 61,1 \\
\hline Entre 5 et 10ans & 55 & 19,3 \\
\hline$\geq 10$ ans & 56 & 19,6 \\
\hline \multicolumn{3}{|c|}{$\begin{array}{l}\text { Facteurs de risque cardiovasculaire } \\
\text { associés }\end{array}$} \\
\hline Surpoids (IMC $\geq 25 \mathrm{~kg} / \mathrm{m}^{2}$ ) & 185 & 64,9 \\
\hline Obésité abdominale & 110 & 38,6 \\
\hline Tabac & 18 & 6,3 \\
\hline Diabète & 50 & 17,5 \\
\hline Hypercholesterolemia LDL* & 119 & 66,5 \\
\hline Syndrome métabolique & 45 & 15,8 \\
\hline \multicolumn{3}{|l|}{ Modalités thérapeutiques } \\
\hline Monothérapie & 86 & 30,2 \\
\hline Bithérapie & 110 & 38,6 \\
\hline Au moins une trithérapie & 89 & 31,2 \\
\hline \multicolumn{3}{|l|}{ Observance thérapeutique } \\
\hline Bonne & 125 & 43,9 \\
\hline Mauvaise & 160 & 56,1 \\
\hline \multicolumn{3}{|l|}{ Contrôle tensionnel } \\
\hline HTA contrôlée & 174 & 61,1 \\
\hline HTA non contrôlée & 111 & 38,9 \\
\hline
\end{tabular}

\section{Etude des complications de l'HTA} $(70,9 \%)$.

Parmi les hypertendus, 202 présentaient au moins une complication de

La fréquence de l'HVG était de $35,7 \%$ à l'ECG et $54,3 \%$ à l'échoDöppler cardiaque. L'insuffisance cardiaque a été notée chez 40 patients (14\%). Il s'agissait d'une insuffisance cardiaque globale chez 28 patients (70\%). La fréquence de l'insuffisance cardiaque était plus élevée chez les hommes que les femmes $(7,7 \%$ vs $6,3 \% ; p=0,025)$.

L'insuffisance coronarienne a été retrouvée dans 48 cas $(16,8 \%)$. L'ischémie myocardique silencieuse était la forme la plus fréquente $(83,3 \%)$.

L'IPS a été mesurée dans 255 cas et l'AOMI retrouvée dans 26 cas $(10,2 \%)$. Selon la classification de Leriche et Fontaine, le stade I était le plus retrouvé $(61,5 \%)$.

L'AVC a été notée dans 9 cas (3.2\%) et le mécanisme était ischémique dans 5 cas.

Le fond d'œil a été réalisé chez 168 patients et il y avait une rétinopathie hypertensive dans 78 cas $(46,4 \%)$. Les stades I et II étaient plus retrouvés avec respectivement $69,2 \%$ et $26,9 \%$. La rétinopathie était plus fréquente chez les hommes que les femmes $(35,5 \%$ vs $23,2 \% ; \mathrm{p}=0,026)$. 
L'atteinte rénale était présente dans 131 cas (46\%). Ces complications rénales étaient la protéinurie isolée (57cas), l'insuffisance rénale isolée (13 cas) et l'association protéinurie avec insuffisance rénale $(59 \mathrm{cas})$.

\section{Etude des facteurs associés à l'HTA compliquée}

En analyse univariée, les facteurs associés à l'existence d'une complication de l'HTA sont présentés dans le tableau II.

Tableau III : Facteurs associés à l'hypertension artérielle (HTA) compliquée chez les patients suivis en milieu cardiologique à Parakou en 2016.

\begin{tabular}{|c|c|c|c|}
\hline & $\begin{array}{l}\text { Total } \\
(\mathrm{N})\end{array}$ & $\begin{array}{l}\text { HTA compliquée } \\
\text { n }(\%)\end{array}$ & $\bar{P}$ \\
\hline Sexe & & & 0,006 \\
\hline Féminin & 174 & $113(64,9)$ & \\
\hline Masculin & 111 & $89(80,2)$ & \\
\hline Age & & & 0,254 \\
\hline$<50$ ans & 106 & $69(65,1)$ & \\
\hline Entre 50 et $70 \mathrm{ans}$ & 155 & $115(74,2)$ & \\
\hline$\geq 70$ ans & 24 & $18(75)$ & \\
\hline Ancienneté de l'HTA & & & 0,068 \\
\hline$<5$ ans & 174 & $118(67,8)$ & \\
\hline Entre 5 et 10 ans & 55 & $46(83,6)$ & \\
\hline$\geq 10$ ans & 56 & $38(67,9)$ & \\
\hline \multicolumn{4}{|l|}{$\begin{array}{l}\text { Facteurs de risque cardiovasculaire } \\
\text { associés }\end{array}$} \\
\hline $\operatorname{IMC}\left(\mathrm{kg} / \mathrm{m}^{2}\right)$ & & & $\mathbf{0 , 0 2 3}$ \\
\hline$\geq 30$ & 88 & $65(73,9)$ & \\
\hline$[25-29,9]$ & 97 & $59(60,8)$ & \\
\hline Obésité abdominale & & & 0,008 \\
\hline Oui & 175 & $134(76,6)$ & \\
\hline Non & 110 & $68(61,8)$ & \\
\hline Tabac & & & 0,780 \\
\hline Oui & 19 & $14(73,7)$ & \\
\hline Non & 266 & $188(70,7)$ & \\
\hline Diabète & & & 0,592 \\
\hline Oui & 50 & $37(74)$ & \\
\hline Non Hypercholestérolémie & 235 & $165(70,2)$ & \\
\hline 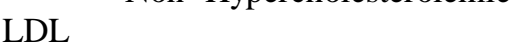 & & & 0,162 \\
\hline Oui & 119 & $91(76,5)$ & \\
\hline Non & 60 & $40(66,7)$ & \\
\hline Syndrome métabolique & & & 0,851 \\
\hline Oui & 28 & $20(71,4)$ & \\
\hline Non & 149 & $109(73,2)$ & \\
\hline Observance thérapeutique & & & 0,001 \\
\hline Bonne & 125 & $75(60)$ & \\
\hline Mauvaise & 160 & $127(79,4)$ & \\
\hline Contrôle tensionnel & & & $\mathbf{0 , 0 2 6}$ \\
\hline HTA contrôlée & 174 & $115(66,1)$ & \\
\hline HTA non contrôlée & 111 & $87(78,4)$ & \\
\hline
\end{tabular}


Une régression logistique incluant le sexe, l'équilibre tensionnel, l'IMC, le tour de taille et l'observance thérapeutique a été réalisée. Seules l'ancienneté de l'HTA $\geq 5$ ans $(\mathrm{OR}=2,9$; IC95\%=1,26-6,64; $\mathrm{p}=0,011)$ et l'inobservance thérapeutique $(\mathrm{OR}=3,1 ; \mathrm{IC} 95 \%=1,5-6,41 ; \mathrm{p}=0,002)$ étaient associées à la survenue de complication de l'HTA.

\section{Discussion}

Dans notre étude, la fréquence de l'HTA en milieu hospitalier cardiologique était de 43,9\% . Cette fréquence est inférieure aux 70,69\% retrouvée par Sonou et al. au CHHU-HKM( Bénin) en 2017 [4] mais corrobore les données de Hajjar et al. aux Etats Unis en 2006 (Sonou, 2017)

Dans ces études, l'HTA reste le principal motif de consultation en milieu cardiologique. Ces fréquences hospitalières sont en général plus élevées que les prévalences en population générale qui varient entre $27,9 \%$ et 32,5\% selon les travaux de Houinato et al. au Bénin en 2012 (Houinato, 2012) et Kimbally-Kaky et al. à Brazzaville en 2004 (Kimbally Kaky, 2004).

La majorité $(70,9 \%)$ des patients suivis était porteuse d'au moins une complication de l'HTA. Ce résultat exprime la méconnaissance de l'hypertension artérielle par les populations qui ne sont vues à l'hôpital qu'en cas d'apparition de symptômes ou de complications. Selon Fourcade et al, la principale raison de cette méconnaissance est le caractère longtemps asymptomatique de l'HTA et l'absence ou l'insuffisance de dépistage systématique (Fourcade, 2007). Cette insuffisance de dépistage est également liée au dysfonctionnement du système sanitaire qui est caractérisé par un nombre insuffisant de centres de santé, le manque d'outils diagnostiques dans les structures sanitaires et la connaissance approximative de l'HTA par les médecins généralistes (Houenassi, 2016). L'HTA est alors prise en charge tardivement et souvent de façon inadéquate et les complications s'installent silencieusement. Mais en plus de ces faits, il faut reconnaitre la sévérité particulière de l'HTA du sujet noir africain qui expose ce dernier à des complications graves (Turner, 2000). Ainsi, l'HTA est habituellement découverte à un moment où le risque cardiovasculaire du patient est élevé voire très élevé avec l'association à d'autres facteurs de risque cardiovasculaire et la multiplicité des retentissements viscéraux (Houenassi, 2013). Plusieurs facteurs expliquent cette vulnérabilité particulière du sujet noir face à l'HTA. Il s'agit de l'hypersensibilité au sodium avec rétention hydrosodée, l'hyperinsulinisme liée à l'obésité, l'hyperréactivité vasculaire en rapport avec la surproduction de l'endothéline 1 et et la chute de l'oxyde nitrique (Nesbitt, 2005).

L'HVG, le plus fréquent des retentissements de l'HTA dans notre étude, constitue un facteur de risque majeur de mortalité cardiovasculaire indépendamment de l'élévation des chiffres tensionnels (Levy, 1990). En 
effet, elle expose le patient à un risque de mort subite (Narayanan, 2014), d'insuffisance coronarienne (Liao, 1995), d'insuffisance cardiaque (Velagaleti, 2014) et de trouble du rythme cardiaque (Levy, 1990). Dans notre étude, elle a été observée chez $54,3 \%$ des patients à l'échographie Döppler cardiaque. Niakara et al. ont rapporté, sur la base de critères échocardiographiques, un taux d'HVG de 53,3\% au Burkina en 2001 (Niakara, 2001). Ces données africaines sont proches des 58\% observés par Cuspidi et al chez les hypertendus en Italie en 2010 (Cuspidi, 2011). Même si le taux d'HVG semble être aussi élevé chez les patients de race blanche que ceux de race noire, la sévérité particulière de l'HVG chez le noir est connue. Havranek et al ont rapporté en 2008 que, en présence d'une HVG, le risque de mortalité à 10ans était 2,3 fois plus élevé chez le noir américain (Havranek, 2008). Complication fréquente de l'HVG chez l'hypertendu, l'insuffisance cardiaque a été observée dans $14 \%$ des cas. A Cotonou en 2017, Sonou et al avaient rapporté un taux plus faible (3,5\%) (Sonou, 2017). ce qui concerne l'insuffisance coronarienne, sa fréquence était de 16,8\%. L'ischémie myocardique silencieuse était la forme prédominante $(83,3 \%)$. Lacourcière et al. au Canada en 2007 avaient noté également dans leur étude que l'ischémie silencieuse était fréquente $(28,9 \%)$ chez les patients hypertendus (Lacourcière, 2007). Mais leur fréquence relativement inférieure à la notre pourrait s'expliquer par la méthode de dépistage utilisée. En effet dans leur étude, l'ischémie myocardique silencieuse a été recherchée par la Tomographie au 99tc qui est une méthode plus spécifique que l'ECG de repos. La fréquence de l'AOMI dans notre étude était de 10,2\%. Ce taux est inférieur aux 41,9\% que nous avions rapportés dans une population de diabétiques suivis à Parakou en 2013 (Codjo, 2016). Cette différence liée à deux raisons principales. Primo, l'HTA est plus fréquente chez le diabétique (Faglia, 2011). Secondo, la technique de dépistage que nous avons utilisé dans la présente étude. En effet, ne disposant pas de Döppler à ultrasons, nous avons dû mesurer la pression systolique à la cheville pour le calcul de l'IPS à l'aide de tensiomètre automatique. Cette technique est peu sensible dans le dépistage de l'AOMI comme l'ont démontré certains travaux (Hamel, 2010). La rétinopathie hypertensive avait été retrouvée chez $28 \%$ de nos patients. Cette fréquence est inférieure à celle retrouvée par Sarcide et al. (Sarcide, 2012) et Devdatta et al. (Devdatta, 2014) qui avaient noté respectivement $66,3 \%$ et $58 \%$ de rétinopathie hypertensive. Cette fréquence moins élevée dans notre étude pourrait se justifier par le fait la plupart de nos patients $(61,1 \%)$ avaient une hypertension équilibrée ; la rétinopathie hypertensive étant la résultante d'une pression artérielle élevée et évoluant sur plusieurs années. 


\section{Limites}

La principale limite de ce travail est son caractère rétrospectif responsable de plusieurs données manquentes. Aussi, de ce fait, des biais de mesure ont pu être introduits dans nos données. Malgré ces insuffisances, nos résultats serviront de base à d'autres études prospectives sur les complications de l'HTA chez le noir africain.

\section{Conclusion}

Ces données confirment que l'hypertension artérielle a une fréquence élevée en cardiologie à Parakou. L'importance des complications observées chez nos patients confirme la gravité de l'hypertension artérielle chez le sujet noir. De plus elle montre que l'hypertendu africain est particulièrement

Des stratégies de santé publique doivent être mises en œuvre pour dépister précocement l'hypertension artérielle, ses complications et garantir une prise en charge optimale aux patients.

\section{Conflit d'intérêt}

Néant

\section{References :}

1. Fourcade L, Paule P, Mafart B (2007). Hypertension artérielle en Afrique subsaharienne. Med Trop, 67(6):559-67.

2. Narayanan K, Reinier K, Teodorescu C, Uy-Evanado A, Chugh H, Gunson K et al (2014). Electrocardiographic versus echocardiographic left ventricular hypertrophy and sudden cardiac arrest in the community. Heart Rhythm, 11(6) : 1040-6.

3. Segbedji GC. Prévalence et aspects sociodémographiques de l'hypertension artérielle chez les adultes de Parakou : cas des quartiers urbains du 1er arrondissement [Thèse de doctorat d'Université en Médecine]. Parakou : Université de Parakou Faculté de Médecine ; 2009. 112p.

4. Sonou DA, Lemone $\mathrm{H}$, Adjagba $\mathrm{P}$, Codjo L, Hounkponou M, Houehanou-Sonou C, Bognon R, Dohou H, Assani S, Tchabi Y, Houenassi M (2017). Etude du risque cardiovasculaire absolu et des complications de l'hypertension artérielle dans une population de patients hypertendus à Cotonou. Journal de la Société de Biologie Clinique du Bénin, 26: 35-40

5. 5-Sahn DJ, DeMaria A, Kisslo J, Weyman A (1978). Recommendations regarding quantitation in M-mode echocardiography: results of a survey of echocardiographic measurements. Circulation, 58:1072-83. 
6. McKee PA, Castelli WP, McNamara PM, Kannel WB (1971). The natural history of congestive heart failure: the Framingham study. NEJM, 285(26), 1441-46.

7. Macfarlane PW. Minnesota coding and the prevalence of ECG abnormalities (2000). Heart, 84:582-4

8. Binaghi M (1995). Rétinopathie hypertensive : Diagnostic, évolution, pronostic. Rev. Prat, 45 : 899-902.

9. Hirsch AT, Haskal ZJ (2006). ACC/AHA 2005 Practice Guidelines for the management of patients with peripheral arterial disease (lower extremity, renal, mesenteric, and abdominal aortic): a collaborative report from the American Association for Vascular Surgery/Society for Vascular Surgery, Society for Cardiovascular Angiography and Interventions, Society for Vascular Medicine and Biology, Society of Interventional Radiology, and the ACC/AHA Task Force on Practice Guidelines (Writing Committee to Develop Guidelines for the Management of Patients With Peripheral Arterial Disease): endorsed by the American Association of Cardio- vascular and Pulmonary Rehabilitation; National Heart, Lung, and Blood Institute; Society for Vascular Nursing; TransAtlantic Inter- Society Consensus; and Vascular Disease Foundation. Circulation, 113(11):e463-e654.

10. Levy AS, Eckardt KU, Tsukamoto Y (2006). Definition and classification of chronic kidney disease : A position statement from kidney disease : improving global outcome (KDIGO). Kidney int. 2005 ; 67 :2089-100. Hajjar I, Kotchen JM, Kotchen TA. Hypertension: trends in prevalence, incidence and control. Annu Rev Public Health, 27:465-90.

11. Hajjar I, Kotchen JM, Kotchen TA (2006). Hypertension : trends in prevalence, incidence and control. Annu Rev Public Health, 27 :46590.

12. Houinato DS, Gbary AR, Houehanou YC, Djrolo F, Amoussou M, Ségnon Agueh J, et al (2012). Prevalence of hypertension and associated risk factors in Benin. Rev Epidemiol Sante Publique, 60 :95-102.

13. Kimbally Kaky G, Gombet T, Bolanda JD, Voumbo Y, Okili B, Ellenga Mbola B, et al (2006). Prevalence of arterial hypertension in Brazzaville. Tropical cardiology, 32(127) :43-6.

14. Houenassi MD, Codjo HL, Dokoui D, Attinsounon AC, Alassani A, Ahoui S et al (2016). Management of arterial hypertension in Cotonou city, Benin: general practitioners' knowledge, attitudes and practice: cardiovascular topics. Cardiovasc J Afr, 23;27(4):e1-e6. doi: 10.5830/CVJA-2015-094. 
15. Turner ST, Boerwinkle E (2000). Genetics of hypertension, target organ complications, and response to therapy. Circulation, 102 (20 Suppl 4) : IV-40-5.

16. Houénassi DM, Tchabi Y, Awanou B, Véhounkpé-Sacca J, Yovo RAD, Sehonou J et al (2013). Évolution du risque cardiovasculaire des patients traités pour HTA à l'hôpital d'instruction des armées de Cotonou. Ann Cardiol Angeiol, 62(1) : 12-6.

17. Nesbitt SD (2005). Hypertension in black patients: Special issues and considerations. Current Hypertension Reports, 7: 244-8

18. Levy D, Garrison R, Savage DD, Kannel WP, Castelli WP et al (1990). Prognostic implications of echocardiographically determined left ventricular mass in the Framingham Heart Study. N Engl J Med, 322:1561-6.

19. Liao Y, Cooper RS, McGee D L, Mensah GA, Ghali JK et al (1995). The relative effects of left ventricular hypertrophy, coronary artery disease and ventricular dysfunction on survival among black adults. JAMA, 273:1592.

20. Velagaleti RS, Gona P, Pencina MJ, Aragam J, Wang TJ, Levy D et al (2014). Left ventricular hypertrophy patterns and incidence of heart failure with preserved versus reduced ejection fraction. Am J Cardiol, 113(1):117-22.

21. Niakara A, Ouédraogo N, Ouandaogo BJ (2001). L'hypertrophie ventriculaire gauche du noir africain hypertendu : étude échocardiographique chez 452 sujets. Ann Cardiol Angeiol, 50(4) :197-201.

22. Cuspidi C, Negri F, Muiesan ML, Capra A, Lonati L, Milan A et al (2011). Prevalence and severity of echocardiographic left ventricular hypertrophy in hypertensive patients in clinical practice. Blood Press, 20(1): 3-9.

23. Havranek EP, Froshaug DB, Emserman CD, Hanratty R, Krantz MJ, Masoudi FA et al (2008). Left ventricular hypertrophy and cardiovascular mortality by race and ethnicity. Am J Med, 121(10): 870-5.

24. Lacourcière Y, Lefebvre J, Poirier L, Coté C, Dumont M (2007). Prévalence de l'ischémie silencieuse chez les hypertendus porteurs d'autres facteurs de risqué cardiovasculaire détecté à l'aide de la tomographie myocardique au 99tc sestamibi. Arch Mal Coeur Vais, 100(8):677-8.

25. Codjo HL, Adoukonou TA, Wanvoegbe A, Dohou H, Bankolé C, Alassani A et al (2016). Prévalence de l'artériopathie oblitérante des membres inférieurs et facteurs associés chez les diabétiques suivis en 
milieu hospitalier à Parakou en 2013. Ann Cardiol Angeiol, 65(4) :260-4.

26. Faglia E (2011). Characteristics of peripheral arterial disease and its relevance to the diabetic population. Int J Low Extrem Wounds, 10(3):152-66. doi: 10.1177/1534734611417352.

27. Hamel JF, Tanguy M, Fanello S (2010). Etude comparative tensiomètre automatique versus doppler à ultrasons dans la mesure de l'index de pression systolique à la cheville. J Mal Vasc, 35(3) :169-74.

28. Sarcide E, Ercan B (2012). Hypertensive Retinopathy: incidence, risk factors, and comorbidities. Clin Exp Hypertens, 34(6):397-401.

29. Devdatta G, Archana C, Vaishali AP, Priyanka RR, Parth KS (2014). Ocular manifestation in hypertensive patients of Jamnagar district. NJIRM, 5(4): 36-40 